\title{
Appraisal of the Current Guidelines for Management of Malignant Left-Sided Colonic Obstruction Using the Appraisal of Guidelines Research and Evaluation II Instrument
}

\author{
Paschalis Gavriilidis $^{a}$ Alan Askari ${ }^{b}$ Nicola de'Angelis ${ }^{c}$ Efstratios P. Gavriilidis ${ }^{d}$ \\ James Wheeler ${ }^{\mathrm{e}}$ Justin Davies ${ }^{\mathrm{e}}$ \\ ${ }^{a}$ Division of Gastrointestinal and Hepato-Biliary-Pancreatic Surgery, Imperial College Healthcare NHS Trust, \\ Hammersmith Hospital, London, UK; ${ }^{b}$ Department of Upper Gastro-Intestinal Surgery, Luton and Dunstable \\ University Hospitals NHS Trust, Luton, UK; ' Department of Digestive Surgery, University Hospital Henri Mondor,

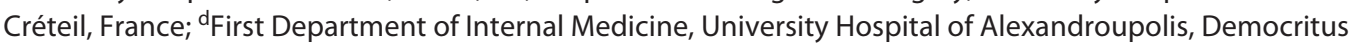 \\ University of Thrace, Alexandroupolis, Greece; ${ }^{e}$ Cambridge Colorectal Unit, Addenbrooke's Hospital, Cambridge \\ University Hospitals NHS Foundation Trust, Cambridge, UK
}

\section{Keywords \\ Clinical practice guidelines · Appraisal of Guidelines for Research and Evaluation II · Acute colonic obstruction}

\begin{abstract}
Introduction: Colorectal cancer (CRC) is the fourth leading cause of death with 1.4 million new cases occurring annually worldwide. High-quality clinical practice guidelines are needed to tailor high-quality individualized treatment. The aim of the present study was to evaluate the methodological quality of the current guidelines for the management of acute malignant left-sided colonic bowel obstruction. Methods: A systematic search of the literature was carried out using electronic databases. The Appraisal of Guidelines for Research and Evaluation (AGREE) II instrument was used to assess the quality of each guideline. Results: Search results returned a total of 14 guidelines appropriate for assessment. Both domain I (scope and purpose) and domain VI (editorial independence) were assessed with the same median score of $83 \%$. The lowest scoring domain was domain V (applicability), scoring only $43 \%$. The 2 guidelines that had the high-
\end{abstract}

est score were the National Institute for Health and Care Excellence (NICE) and Scottish Intercollegiate Guidelines Network (SIGN), each scoring 100\%. However, there were significant variations in terms of quality. The NICE and New Zealand guidelines were voted unanimously for use unchanged, whilst 8 other guidelines were voted for use with modifications. Conclusion: Variation in guideline quality in CRC is a concern despite some clearly excellent published guidelines. All guidelines score poorly when it comes to describing how the guidelines could be applied. Lack of patient participation in guideline development is also a shortcoming that requires urgent redress.

(c) 2021 S. Karger AG, Basel

\section{Introduction}

Colorectal cancer (CRC) is the second most common cancer in women and third in men and the fourth leading cause of death worldwide; every year, 1.4 million new cases occur $[1,2]$. Two thirds of CRCs occur in high-income countries and there is an increase in prevalence due to an

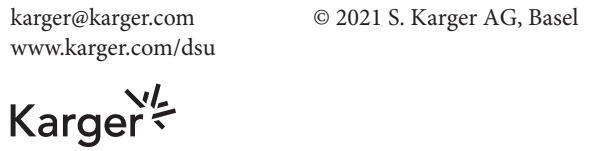

Paschalis Gavriilidis

Division of Gastrointestinal and Hepato-Biliary-Pancreatic Surgery Imperial College Healthcare NHS Trust, Hammersmith Hospital 72 Du Cane road, London W12 0HS (UK)

pgavrielidis@yahoo.com 
ageing population. However, a decrease in mortality rate of around 35\% over the last 2 decades has been reported, possibly because of earlier diagnosis through screening programmes and better multimodality treatment approaches [3-5]. In 2011, the Institution of Medicine (US) defined clinical practice guidelines (CPGs) as "Clinical practice guidelines are statements that include recommendations intended to optimize patient care that are informed by a systematic review of the evidence and an assessment of benefits and harms of alternative care options" [6].

So far, more than 40 tools have been described for appraisal of CPGs; the latest is the Appraisal of Guidelines for Research and Evaluation (AGREE II) [7]. The AGREE II instrument was validated internationally and endorsed by several institutions and organizations including the WHO Advisory Committee on Health Research and by many guidelines' development teams $[8,9]$. Further information is available on the AGREE website (www. agreetrust.org).

The aim of the present study was to evaluate the quality of current guidelines, with a primary focus on management of acute malignant left-sided colonic obstruction, using the AGREE II instrument. The present study focused on methodological analysis and did not analyse the recommended practices.

\section{Methods}

Our rationale for selecting malignant left-sided colonic obstruction as the main focus of this study was due to several clinically relevant factors. Firstly, left-sided malignant colonic obstruction is a common occurrence in daily surgical practice. Secondly, the management options available in the treatment of left-sided malignant colonic obstruction are perhaps more numerous than right sided, allowing a wider assessment of guidelines. Finally, patients presenting with left-sided malignant colonic obstruction may pose physiological challenges and surgery is often high risk and associated with significant morbidity and mortality, making it even more crucial that the quality of guidelines in this area is high.

Search Strategy and Guideline Selection

Two of the authors (P.G. and A.A.) independently carried out electronic searches of the existing literature using the following databases: Cochrane, PubMed, EMBASE, and Google Scholar using the keywords: colonic, cancer, obstruction, and guidelines. No restrictions were placed on guidelines from any region or country, but searches were limited to those guidelines published in the English language and within the last 20 years. The PRISMA (Preferred Reporting Items for Systematic Reviews and Meta-Analyses) [10] criteria were used to demonstrate search results and reasons for exclusion. Manual searches of retrieved manuscripts were also carried out to ensure that no further guidelines were missed.
Data Extraction and Guideline Appraisal

After independent evaluation of the CPGs by 2 authors (P.G. and A.A.), the following data were extracted: country of origin, year of publication, development and/or revision committee, evaluation measures, and funding sources. Evidenced-based CPGs in the English language pertaining to the management of acute malignant left-sided colonic obstruction were included.

The AGREE II tool is a validated checklist that is designed to assess the quality of clinical guidelines. It comprises 23 items divided into 6 domains: scope and purpose, stakeholder involvement, rigour of development, clarity of presentation, applicability, and editorial independence. Assessors use the checklist to rate the quality of each clinical guideline across all 23 items using an online platform. An aggregate score is then provided for each domain for each clinical guideline. Each of the 6 domains assesses a different aspect of the guideline. For example, domain I concentrates on how well guidelines set out their aims and who their target audience/users are whilst domain II assesses whether relevant key healthcare workers and patient groups have been involved in the development of the guidelines. Domains III and IV focus on the methodology the guideline has used in formulating their recommendations, that is, its search strategy, inclusion and assessment of the evidence available, and whether the guideline's key recommendations are presented in a clear, user friendly manner. Domain $\mathrm{V}$ assesses how good the guidelines are in giving advice to clinicians on implementation, including the resources and staffing required. Domain VI ensures that the persons writing the guidelines were free from any conflict of interest and were not unduly influenced by any external organization.

For further details regarding the criteria used to describe and evaluate the 6 domains and the 23 consisted items, please see online suppl. Table 1 (for all online suppl. material, see www.karger. com/doi/10.1159/000514446): AGREE reporting checklist. After undergoing online training (www.agreetrust.org) to ensure appraisal standardization, 4 appraisers (P.G., A.A., N.A., and E.G.), as recommended by the AGREE II consortium, evaluated the guidelines, independently using the AGREE II tool (September 2013 version). As per the AGREE II manual, discrepancies of more than 2 standard deviations were resolved through dialogue. Authors had the ability to change their entry after group discussion. Domain scores were calculated with the following formula.

\section{Results}

The search result yielded a total 110 manuscripts, of which 15 were excluded as they were duplicates. Of the remaining 95, 81 were excluded as they were not relevant and did not meet the inclusion criteria, leaving a total of 14 guidelines [7, 11-23] remaining for appraisal (Fig. 1). The majority of the guidelines presented relevant information on the epidemiological, diagnostic, and treatment algorithms (Table 1). Across the 6 domains, the highest scoring were domains I (scope \& purpose) and domain VI (editorial independence), with both scoring a median of $83 \%$ (Fig. 2). Domain IV (clarity of presentation) also 
Fig. 1. PRISMA flowchart outlining the search results for guidelines. AGREE, Appraisal of Guidelines for Research and Evaluation.

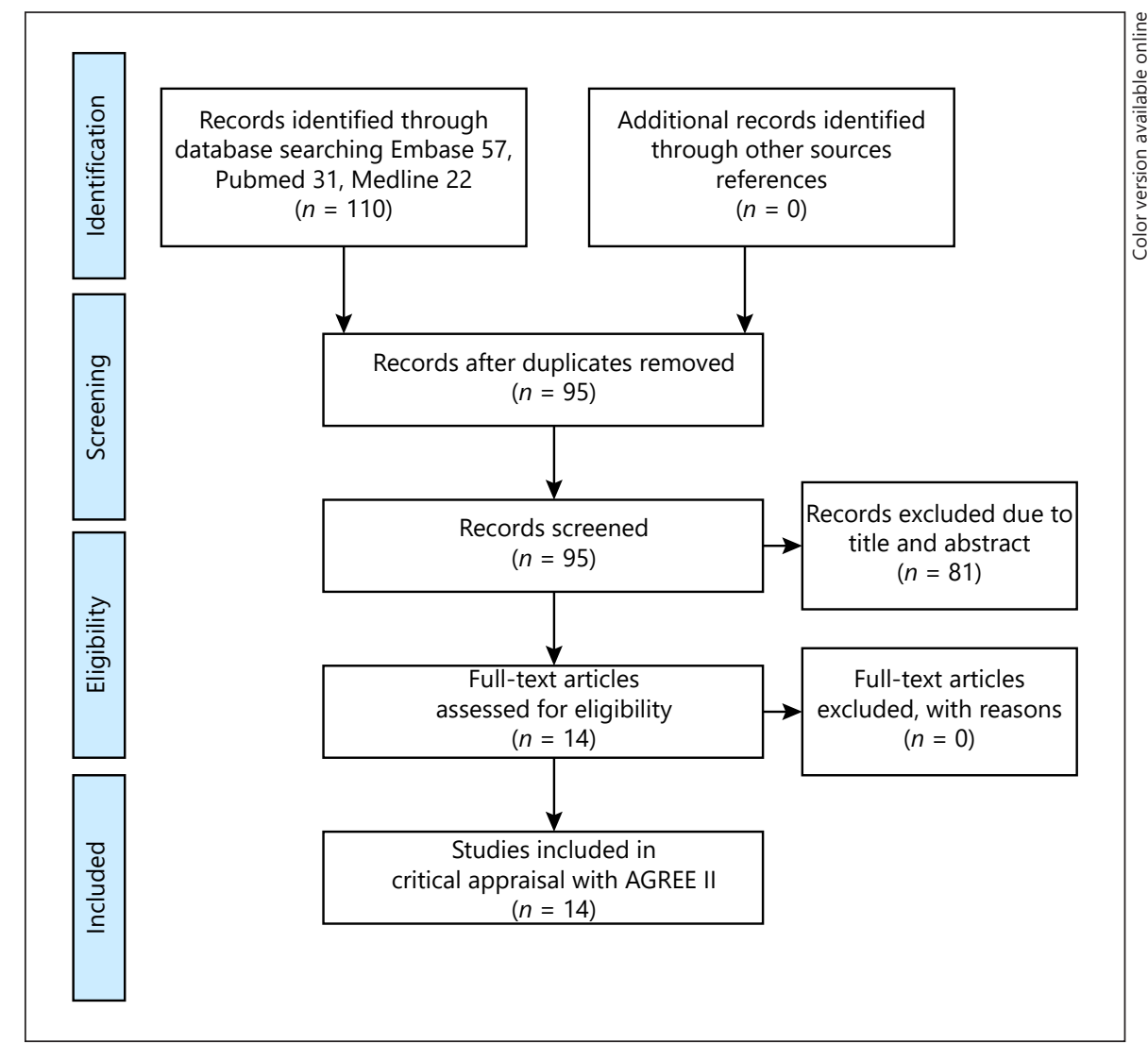

Table 1. Study characteristics

\begin{tabular}{llllll}
\hline Developer & Year & Content & Guideline review & Scoring system & Evidence based \\
\hline NICE & 2020 & E, D\&T, and FU & Yes & GRADE & NICE guidelines-methodology expert panel \\
GGPO & 2019 & E, D\&T, and FU & Yes & GRADE & Systematic literature review and expert panel \\
Australian & 2018 & E, D\&T, and FU & Yes & GRADE & Systematic literature review and expert panel \\
ACPGBI & 2017 & E, D\&T, and FU & Members of ACPGBI & GRADE & Systematic literature review and expert panel \\
ASCRS & 2017 & E and D\&T & NR & GRADE & Expert panel \\
WSES & 2017 & E and D\&T & Yes & Levels of evidence & Expert panel \\
SIGN & 2016 & E, D\&T, and FU & NR & GRADE & Expert panel \\
EAST & 2015 & E and D\&T & EASL reviewers & Oxford + GRADE & Systematic literature review and expert panel \\
ASGBI & 2014 & E and D\&T & NR & GRADE & NR \\
ESGE & 2014 & E, D\&T, and FU & ESGE & GRADE & Systematic literature review and consensus panel \\
EURECCA & 2013 & E, D\&T, and FU & Yes & Oxford + GRADE & Systematic literature review \\
NCCN & 2011 & E, D\&T, and FU & Members of NCCN & Oxford + GRADE & Systematic literature review and expert panel \\
NZGG & 2011 & E, D\&T, and FU & NR & GRADE & Expert panel \\
ASGE & 2010 & E and D\&T & NR & NR & Expert panel \\
\hline
\end{tabular}

NICE, National Institute for Health and Care Excellence; GGPO, German Guideline Program in Oncology; ACPGBI, Association of Coloproctology of Great Britain \& Ireland; ASCRS, American Society of Colon and Rectal Surgeons Committee; WSES, World Society of Emergency Surgery; SIGN, Scottish Intercollegiate Guidelines Network; EAST, East Association for the Surgery of Trauma; ASGBI, Association of Surgeons of Great Britain and Ireland; ESGE, European Society of Gastrointestinal Endoscopy; EURECCA, European Consensus Conference Colon \& Rectum; NCCN, National Comprehensive Cancer Network; NZGG, New Zealand Guidelines Group; ASGE, American Society of Gastrointestinal Endoscopy; GRADE, Grade of Recommendations Assessment, Development, \& Evaluation; E, epidemiology, D\&T, diagnosis \& treatment; FU, follow-up; NR, not reported. 


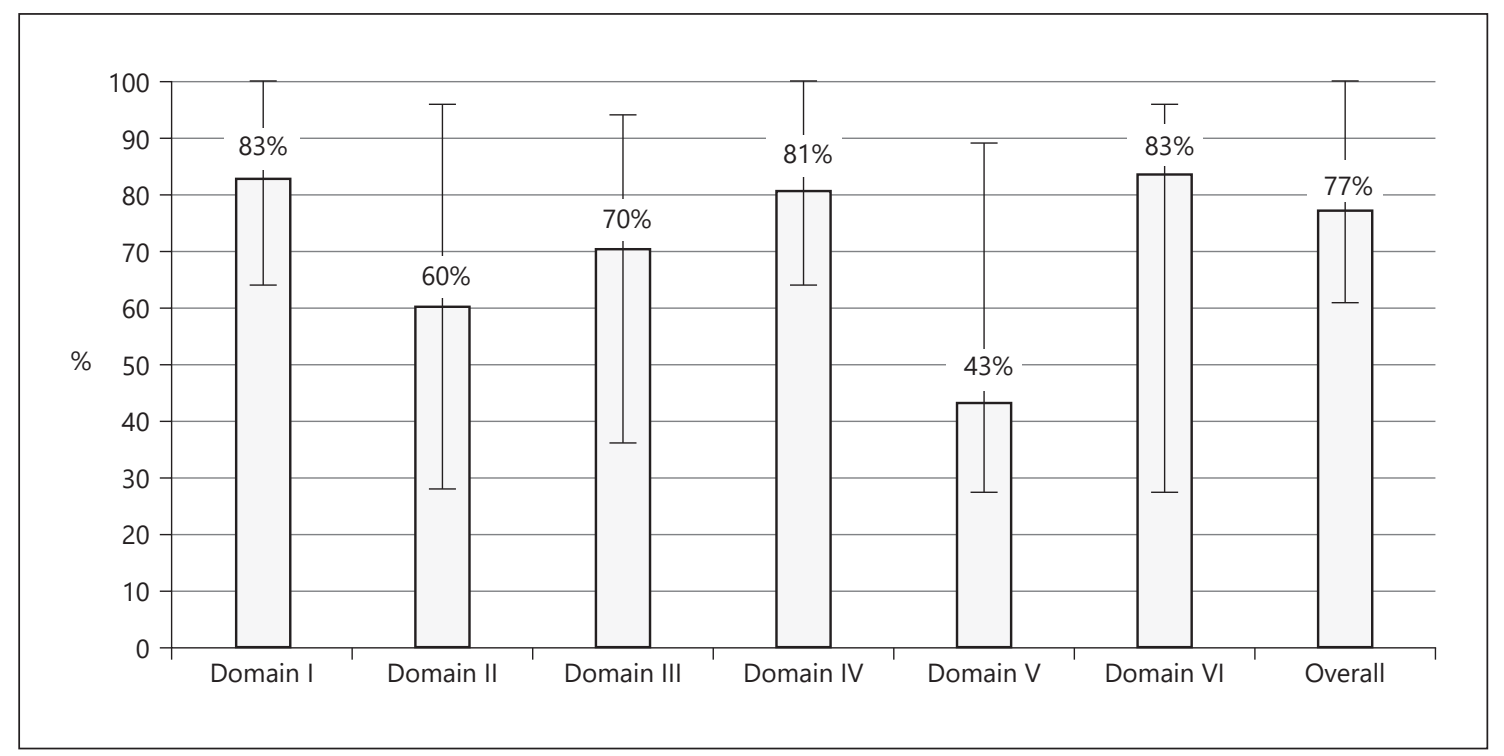

Fig. 2. Median scores for each domain for all guidelines pooled together. Error bars denote the range of scores: lowest and highest.

Table 2. Outcome of votes by the 4 appraisers

\begin{tabular}{|c|c|c|c|c|c|c|c|c|c|c|c|c|c|c|}
\hline Recommend? & ACPGBI & ASCRS & ASGBI & ASGE & Australia & EAST & ESGE & EURECCA & GGPO & $\mathrm{NCCN}$ & NICE & NZGG & SIGN & WSES \\
\hline Yes & 0 & 1 & 1 & 0 & 1 & 0 & 1 & 1 & 2 & 1 & 4 & 4 & 2 & 2 \\
\hline Yes, with modification & 4 & 2 & 3 & 3 & 3 & 3 & 3 & 3 & 2 & 2 & 0 & 0 & 2 & 2 \\
\hline No & 0 & 1 & 0 & 1 & 0 & 1 & 0 & 0 & 0 & 1 & 0 & 0 & 0 & 0 \\
\hline
\end{tabular}

Outcome of votes by the 4 appraisers regarding whether guidelines would be recommended for clinical use. ACPGBI, Association of Coloproctology of Great Britain \& Ireland; ASCRS, American Society of Colon and Rectal Surgeons Committee; ASGBI, Association of Surgeons of Great Britain and Ireland; ASGE, American Society of Gastrointestinal Endoscopy; EAST, East Association for the Surgery of Trauma; ESGE, European Society of Gastrointestinal Endoscopy; EURECCA, European Consensus Conference Colon \& Rectum; GGPO, German Guideline Program in Oncology; NICE, National Institute for Health and Care Excellence; NZGG, New Zealand Guidelines Group; SIGN, Scottish Intercollegiate Guidelines Network; WSES, World Society of Emergency Surgery.

scored well (median $81 \%$, range $64-100 \%$ ). Of the remaining domains, domain II (stakeholder involvement) and domain III (rigour of development) scored lower with a median score of $60 \%$ (range $28-96 \%$ ) and $70 \%$ (range 36-94\%) respectively. Domain V (applicability) scored particularly poorly with a median score of $43 \%$ (range 27-89\%).

Of the various different guidelines, those from SIGN (Scottish Intercollegiate Guidelines Network), NZGG (New Zealand Guidelines Group), and the United Kingdom-based NICE (National Institute for Health \& Care Excellence) scored the highest consistently (Fig. 3). The NICE guidelines scored the highest in domains I, II, III,
Fig. 3. Results of appraisal per domain for each of the guidelines. NICE, National Institute for Health and Care Excellence; GGPO, German Guideline Program in Oncology; ACPGBI, Association of Coloproctology of Great Britain \& Ireland; ASCRS, American Society of Colon and Rectal Surgeons Committee; WSES, World Society of Emergency Surgery; SIGN, Scottish Intercollegiate Guidelines Network; EAST, East Association for the Surgery of Trauma; ASGBI, Association of Surgeons of Great Britain and Ireland; ESGE, European Society of Gastrointestinal Endoscopy; EURECCA, European Consensus Conference Colon \& Rectum; NCCN, National Comprehensive Cancer Network; NZGG, New Zealand Guidelines Group; ASGE, American Society of Gastrointestinal Endoscopy.

(For figure see next page.) 

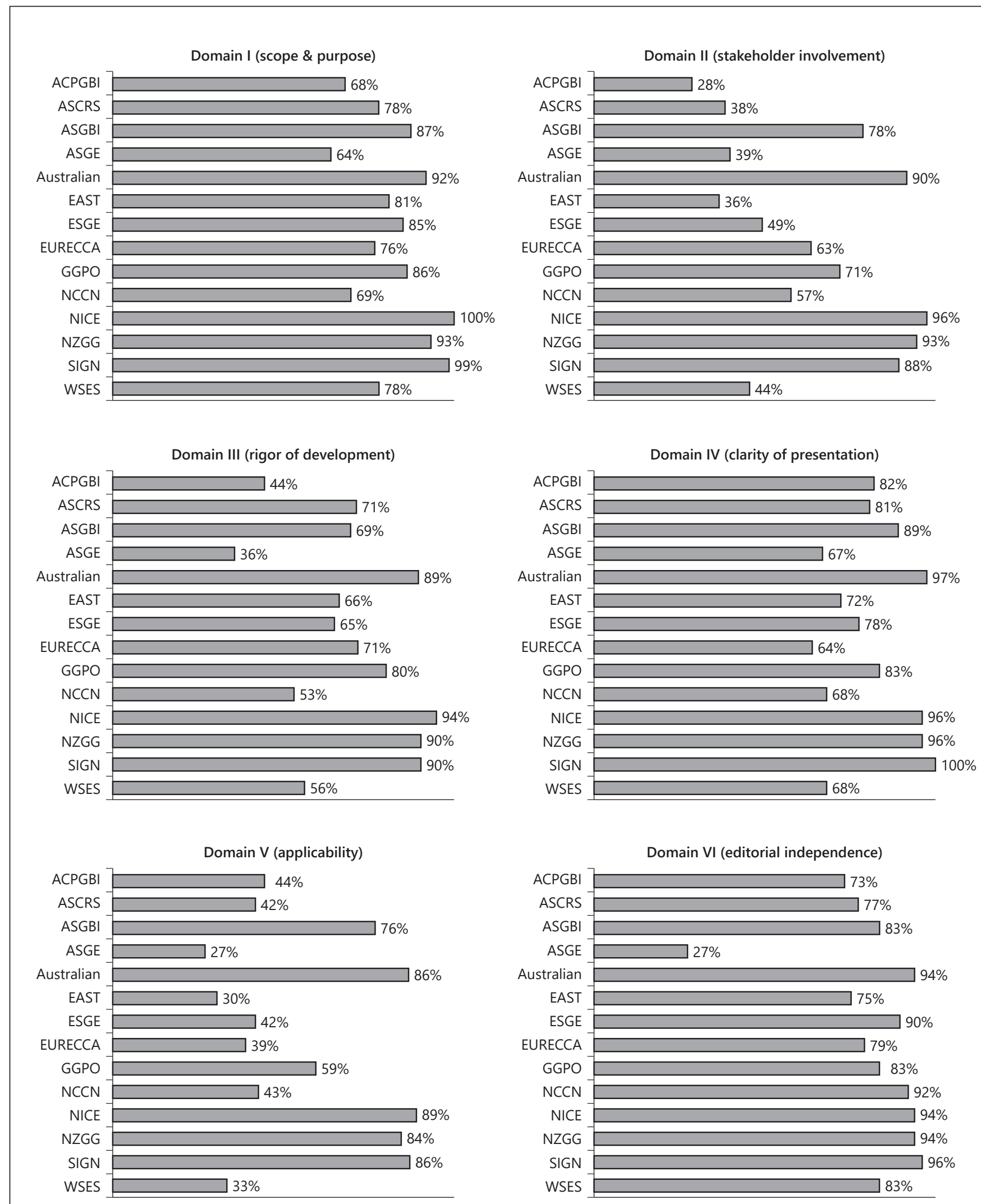
and V whilst SIGN scored highest in IV and VI. Conversely, the lowest scoring guideline was the ASGE (American Society of Gastrointestinal Endoscopy) which had a median score of $38 \%$ across all of the domains.

One of the AGREE II checklist questions is whether appraisers would use the guideline in their daily clinical practice. Only 2 of the guidelines (NZGG and NICE) scored a unanimous $4 / 4$ votes from the appraisers. SIGN, WSES (World Society of Emergency Surgery), and GGP (German Guideline Program in Oncology) scored 2 "Yes" votes and 2 "Yes with Modification" (Table 2).

\section{Discussion}

This study is the first appraisal of the methodological quality of guidelines in CRC using the AGREE II instrument. The overall quality of guidelines was good, with a median score of $77 \%$. This compares relatively favourably with similar appraisals of some other clinical guidelines in hepatobiliary conditions $[24,25]$. As a general rule, many guidelines struggle to score respectable marks irrespective of speciality. There is, however, evidence that the quality of guidelines is improving over time as a systematic review of 626 guidelines across an array of subspecialities has demonstrated [9].

One of the areas in which guidelines appear to consistently fall down is in applicability (domain V) and, to some extent, stakeholder involvement (domain II) whereas they tend to score well in areas of aim and scope (domain I), rigour of development (domain IV), and editorial independence (domain VI). What this ultimately implies is that whilst most guidelines are reasonable in explaining their aims, outlining the way they have examined the evidence, the rationale behind their recommendations, and the transparency of their editorial team in terms of conflicts of interest is often poorly articulated. They often lack any significant involvement of patient advocacy groups and little or no explanation or idea as to how to actually implement the guideline. As such, most guidelines, as has been historically demonstrated by several AGREE assessments [26], lack helpful instructions on their practical application. Unfortunately, guidelines for the management of malignant left-sided colonic obstruction appear little better, scoring low in the applicability domain (domain V), with some scoring as low as 27\% (ASGE guidelines).

Of note, the guidelines from the United Kingdom (NICE and SIGN) and New Zealand (NZGG) performed fairly well and scored between 84 and $89 \%$ in the applicability domain. Some of the reasons behind this higher scoring may be explained in describing how consideration of types of facilitators and barriers in guideline implementation were addressed in the cohort of patients with peritoneal carcinomatosis being considered for cytoreductive surgery and hyperthermic intraperitoneal chemotherapy. Because the evidence was mixed, feedback was sought from key stakeholders including representatives from centres with specific expertise and skills to deliver the recommended care so that more patients can have potentially curative treatment and to avoid centres offering treatment without having the necessary training and resources. Furthermore, additional materials such as guideline summary documents, links to checklists, algorithms, how-to manuals, tools to capitalize on guidelines facilitators, and outcomes of pilot test and lessons learnt were outlined. In addition, health economists were integral members of the guideline development panel and contributed in terms of assessment of specific surgical procedure costs per treatment course with calculation of quality-adjusted life years. Finally, the audit criteria to assess guideline implementation and adherence to recommendations were described, along with operational definitions of how the criteria should be measured.

Furthermore, omission of patient advocacy groups or certain relevant healthcare and allied care professions deprives the guidelines of several important attributes; legitimacy and depth and breadth of view. Public or patient representation is particularly important as this may offer important insights and clarity from a patient perspective. This will likely add great value to the quality of guidelines, making them more practically useful in daily clinical practice. Not only is the involvement of patient's preference morally and medicolegally sensible, there is strong evidence that such patient representation in guideline formulation reduces the adoption of unhelpful or outdated clinical practice [27-29]. The NICE and SIGN guidelines in particular made special mention of the inclusion of patient representatives in their guideline formulation. However, these appear to be the exception rather than the rule, as a recent study reported that CRC guidelines seldom include the patient perspective [30].

The issue is not just with variable rating across domains. The quality of guidelines also varies according to country of origin [31], by clinical speciality, and even different conditions within that same subspeciality [32]. This is an issue that requires urgent redress, not least because this discrepancy in quality leaves clinicians in a difficult conundrum when faced with choosing local versus 
international guidelines. Certainly, guidelines that are practical to implement and simple to understand are more likely to be implemented $[33,34]$.

Despite the limitations of many guidelines, there are important domains where they tend to score well; rigour of development is one example. In the present study, the median score was $70 \%$ across all studies for this domain. Perhaps the main reason why guidelines often score well in this domain is that they universally use some form of validated appraisal system to examine the evidence on which they base their recommendations. In this study, the GRADE (Grade of Recommendations Assessment, Development, \& Evaluation) and Oxford systems were most commonly used. The only exception was the ASGE guidelines, which did not report the use of any particular assessment method and this is the likely explanation for its low score (36\%). The use of grading and appraisal tools is important in formulating guidelines as it ensures a level of uniformity and standardization in assessing evidence to be included in the guideline irrespective of the body, organization, or country from which the guideline hails. In short, it assures a "level playing field" in scrutinizing the evidence.

Guideline quality aside, it is perhaps also important to discuss the use of any guideline. Whilst the benefits of guideline use are well recognized, namely, in providing clinical advice and policy based on the best evidence available, standardizing care, helping to deliver efficient and high-quality care, they do also suffer from certain limitations. One of the greatest worries regarding guidelines is that the information a recommendation is based on could be flawed or incorrect. The methodology and rigour of evidence assessment varies greatly between guidelines and it is conceivable that evidence is overlooked or misinterpreted by guideline authors and editors [29]. To make matters worse, guidelines can potentially lead to a "herd mentality" approach whereby clinicians follow a subscribed protocol or care algorithm irrespective of the patient's unique personal and healthcare needs. In a refreshingly candid editorial, Fred warns (with poignant examples) against the dangers of a certain type of "mental inertia" leading to misdiagnosis or inappropriate treatment of patients due to unreflective and uncritical application of guidelines without considering individual patient characteristics [35]. Finally, guidelines may become out of date between their planned revisions, as new trials are published and evidence accumulates.

This study used the validated AGREE II checklist which has a long history of use as the main assessment tool for CPGs. However, that does not mean that the study is free of limitations. Although blinded to each oth- er's assessments, an element of bias could still have affected the judgement of the appraisers. It is possible that clinicians awarded higher marks to guidelines from their native region or ones that they were already relatively familiar with. Guidelines from well-known organizations may have been awarded undeserving high marks based on reputation, and equally, lesser well-known organizations may have had their guidelines placed under greater scrutiny. There are also inherent issues with the AGREE instrument itself. In particular, the divisions of the domains of the AGREE checklist is polychotomous; this principal characteristic gives equal importance to all the domains; whereas it is unclear whether all domains contribute equally in the construction of high-quality guidelines [28].

The main finding of this study is that clinical guidelines for left-sided malignant colonic obstruction are of an extremely varied quality. Future guidelines should aim to include increased patient representation to help develop guidelines that are able to provide clear and specific instructions on how to best implement the guidance in everyday clinical practice. We, therefore, recommend that all future guidelines be formulated with the domains of the AGREE II checklist in mind, to allow greater inclusion of relevant clinical and patient groups and more specifically, useful advice on how to best implement the advice outlined in the guidelines.

\section{Acknowledgements}

This article does not include any non-author contributors to acknowledge.

\section{Statement of Ethics}

This study does not contain any studies with human participants or animals performed by any of the authors.

\section{Conflict of Interest Statement}

All named authors hereby declare that they have no conflict of interest to disclose. R.J. Davies was topic advisor for the NICE Colorectal Cancer Guidelines (NG 151) but was not one of the authors performing the literature search or guideline appraisal.

\section{Funding Sources}

This study did not receive any funding. 


\section{Author Contributions}

P.G.: study concept and design, acquisition of data, analysis and interpretation of data, drafting the manuscript, appraisal of the guidelines, statistical analysis, and critical revision of the manuscript for important intellectual content. A.A.: acquisition of data, analysis and interpretation of data, drafting the manuscript, appraisal of the guidelines, statistical analysis, and critical revision of the manuscript for important intellectual content. N.D.A.: analysis and interpretation of data, appraisal of the guidelines, and critical revision of the manuscript for important intellectual content. E.P.G.: analysis and interpretation of data, appraisal of the guidelines, and critical revision of the manuscript for important intellectual content. J.W.: analysis and interpretation of data, drafting the manuscript, statistical analysis, and critical revision of the manuscript for important intellectual content. J.D.: study concept and design, acquisition of data, analysis and interpretation of data, drafting the manuscript, statistical analysis, critical revision of the manuscript for important intellectual content, and study supervision.

\section{References}

1 Arnold M, Sierra MS, Laversanne M, Soerjomataram I, Jemal A, Bray F. Global patterns and trends in colorectal cancer incidence and mortality. Gut. 2017;66(4):683-91.

2 Torre LA, Siegel RL, Ward EM, Jemal A. Global cancer incidence and mortality rates and trends: an update. Cancer Epidemiol Biomarkers Prev. 2016;25(1):16-27.

3 Bray F, Ferlay J, Soerjomataram I, Siegel RL, Torre LA, Jemal A. Global cancer statistics 2018: GLOBOCAN estimates of incidence and mortality worldwide for 36 cancers in 185 countries. CA Cancer J Clin. 2018;68(6):394424.

4 Maule M, Merletti F. Cancer transition and priorities for cancer control. Lancet Oncol. 2012;13(8):745-6.

5 Ferlay J, Colombet M, Soerjomataram I, Mathers C, Parkin DM, Piñeros M, et al. Estimating the global cancer incidence and mortality in 2018: GLOBOCAN sources and methods. Int J Cancer. 2019;144(8):1941-53.

6 Graham R, Mancher M, Wolman DM, Greenfield S, Steinberg E, editors. Clinical practice guidelines we can trust. Washington, D.C.: National Academies Press; 2011.

7 Brouwers MC, Kho ME, Browman GP, Burgers JS, Cluzeau F, Feder G, et al. AGREE II: advancing guideline development, reporting, and evaluation in health care. Prev Med. 2010; 51(5):421-4.

8 Coroneos C, Voineskos S, Cornacchi S, Goldsmith C, Ignacy T, Thoma A. Users' guide to the surgical literature: how to evaluate clinical practice guidelines. Can J Surg. 2014;57:280-6.

9 Alonso-Coello P, Irfan A, Solà I, Gich I, Delgado-Noguera $M$, Rigau $D$, et al. The quality of clinical practice guidelines over the last two decades: a systematic review of guideline appraisal studies. Qual Saf Health Care. 2010; 19(6):e58.

10 Moher D, Liberati A, Tetzlaff J, Altman DG; PRISMA Group. Preferred reporting items for systematic reviews and meta-analyses: the PRISMA statement. PLoS Med. 2009;6: e1000097.

11 German Cancer Society. German cancer aid. S3- guideline colorectal cancer, long version 2.1. 2019. Available from: www.leitlinienprogramm-onkologie.de/leitlinien/kolorektaleskarzinom/ Accessed 2020 May 7.
12 Benson AB, Arnoletti JP, Bekaii-Saab T, Chan E, Chen YJ, Choti MA, et al. Colon cancer. J Natl Compr Canc Netw. 2011;9(11):1238-90.

13 New Zealand Guidelines Group. Clinical practice guidelines for the management of early colorectal cancer. 2011. Available from: https:// www.health.govt.nz Accessed 2020 May 7.

14 ASGE Standards of Practice Committee, Harrison ME, Harrison ME, Anderson MA, Appalaneni V, Banerjee S, Ben-Menachem T, et al. The role of endoscopy in the management of patients with known and suspected colonic obstruction and pseudo-obstruction. Gastrointest Endosc. 2010;71(4):669-79.

15 Pisano M, Zorcolo L, Merli C, Cimbanassi S, Poiasina E, Ceresoli M, et al. 2017 WSES guidelines on colon and rectal cancer emergencies: obstruction and perforation. World J Emerg Surg. 2018;13:36.

16 Cancer Council Australia Colorectal Cancer Guidelines Working Party. COLMING5: what are the benefits of stenting or colostomy vs acute resection with primary anastomosis in acute obstruction due to left-sided colon or rectal carcinoma? Cancer council of Australia. 2018. Available from: https://wiki.cancer. org.au Accessed 2020 May 7.

17 Vogel JD, Eskicioglu C, Weiser MR, Feingold DL, Steele SR. The American society of colon and rectal surgeons clinical practice guidelines for the treatment of colon cancer. Dis Colon Rectum. 2017;60:999-1017.

18 Moran B, Cunningham C, Singh T, Sagar P, Bradbury J, Geh I, et al. Association of coloproctology of Great Britain \& Ireland (ACP$\mathrm{GBI}$ ): guidelines for the management of cancer of the colon, rectum and anus (2017): surgical management. Colorec Dis. 2017; 19(Suppl 1):18-36.

19 Scottish Intercollegiate Guidelines Network (SIGN). Diagnosis and management of colorectal cancer. 2011.

20 Ferrada P, Patel MB, Poylin V, Bruns BR, Leichtle SW, Wydo S, et al. Surgery or stenting for colonic obstruction: a practice management guideline from the Eastern association for the surgery of trauma. J Trauma Acute Care Surg. 2016;80(4):659-64.
21 The Royal College of Surgeons of England. Commissioning guide: emergency general surgery (acute abdominal pain). 2014. Available from: https://www.rcseng.ac.uk/Accessed 2020 May 7.

22 van Hooft JE, van Halsema EE, Vanbiervliet G, Beets-Tan RG, DeWitt JM, Donnellan F, et al. Self-expandable metal stents for obstructing colonic and extracolonic cancer: European society of gastrointestinal endoscopy (ESGE) clinical guideline. Gastrointest Endosc. 2014;80(5):747-75.

23 van de Velde CJH, Boelens PG, Borras JM, Coebergh JW, Cervantes A, Blomqvist L, et al. EURECCA colorectal: multidisciplinary management: European consensus conference colon \& rectum. Eur J Cancer. 2014;50: 1.e1-1.e34.

24 Gavriilidis P, Roberts KJ, Askari A, Sutcliffe RP, Huo TL, Liu PH, et al. Evaluation of the current guidelines for resection of hepatocellular carcinoma using the Appraisal of Guidelines for Research and Evaluation II instrument. J Hepatol. 2017;67(5):991-8.

25 Gavriilidis P, Askari A, Roberts KJ, Sutcliffe RP. Appraisal of the current guidelines for management of cholangiocarcinoma-using the Appraisal of Guidelines Research and Evaluation II (AGREE II) Instrument. Hepatobiliary Surg Nutr. 2020;9(2):126-35.

26 O'Donoghue KJM, Reed RD, Knight SR, O'Callaghan JM, Ayaz-Shah AA, Hassan S, et al. Systematic review of clinical practice guidelines in kidney transplantation. BJS Open. 2017;1:97-105.

27 Hämeen-Anttila K, Komulainen J, Enlund H, Mäkelä M, Mäkinen E, Rannanheimo P, et al Incorporating patient perspectives in health technology assessments and clinical practice guidelines. Res Social Adm Pharm. 2016; 12(6):903-13.

28 Krahn M, Naglie G. The next step in guideline development: incorporating patient preferences. JAMA. 2008;300(4):436-8.

29 Woolf SH, Grol R, Hutchinson A, Eccles M, Grimshaw J. Clinical guidelines: potential benefits, limitations, and harms of clinical guidelines. BMJ. 1999;318(7182):527-30. 
30 Selva A, Sanabria AJ, Niño de Guzman E, Ballesteros M, Selva C, Valli C, et al. Colorectal cancer guidelines seldom include the patient perspective. J Clin Epidemiol. 2019;116: 84-97.

31 Choi T-Y, Choi J, Lee JA, Jun JH, Park B, Lee MS. The quality of clinical practice guidelines in traditional medicine in Korea: appraisal using the AGREE II instrument. Implement Sci. 2015; 10:104.
32 Bhatt M, Nahari A, Wang PW, Kearsley E, Falzone N, Chen S, et al. The quality of clinical practice guidelines for management of pediatric type 2 diabetes mellitus: a systematic review using the AGREE II instrument. Syst Rev. 2018;7(1):193.

33 Tong A, Chapman JR, Wong G, de Bruijn J, Craig JC. Screening and follow-up of living kidney donors: a systematic review of clinical practice guidelines. Transplantation. 2011; 92(9):962-72.
34 Acuna SA, Huang JW, Scott AL, Micic S, Daly C, Brezden-Masley C, et al. Cancer screening recommendations for solid organ transplant recipients: a systematic review of clinical practice guidelines. Am J Transplant. 2017; 17(1):103-14.

35 Fred HL. Elephant medicine revisited. Tex Heart Inst J. 2008;35(4):385-7. 